\title{
Novel, Highly-Parallel Software for the Online Storage System of the ATLAS Experiment at CERN: Design and Performances
}

Tommaso Colombo ${ }^{a, b} \quad$ Wainer Vandelli ${ }^{b}$

a Università degli Studi di Pavia

$$
{ }^{b} \text { CERN }
$$

IEEE Real-Time Conference, 12 June 2012 


\section{The ATLAS Trigger \& DAQ System}

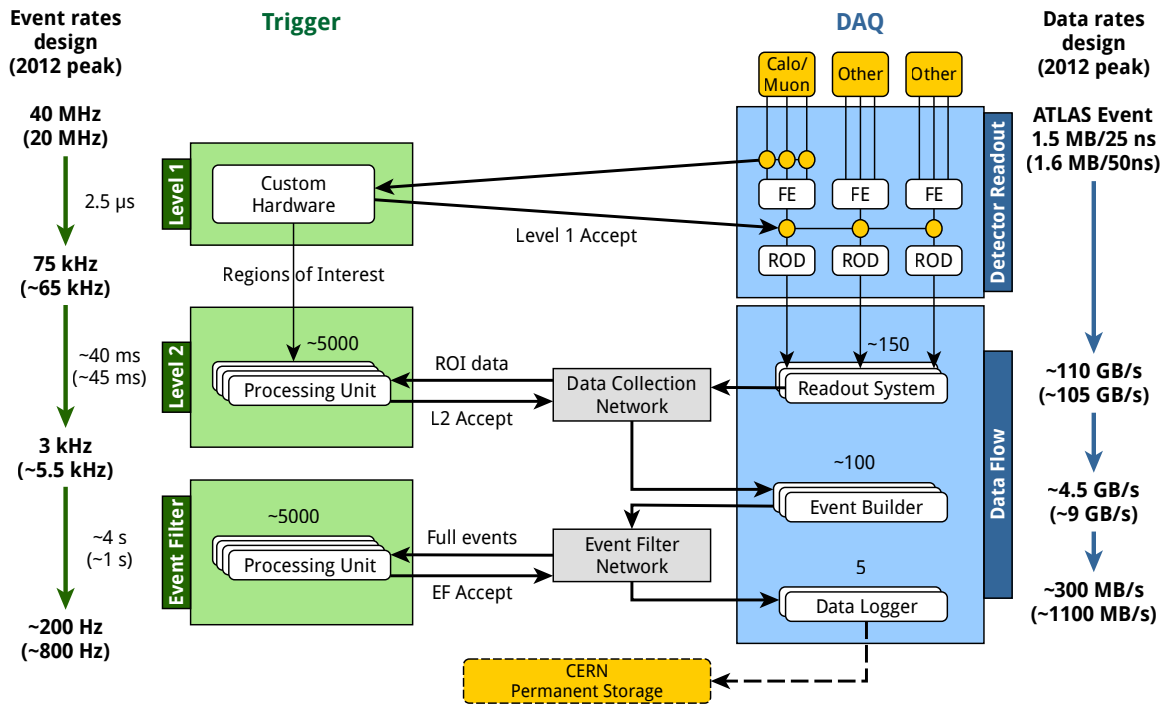




\section{The current Data Logging system: overview}

\section{Purpose}

5 PCs receive data from the Event Filter system and write it to local disks. Each event is:

- analyzed to determine the "tags" applied by the Event Filter trigger

- processed (e.g. compressed)

- written to appropriate file(s) according to the tags

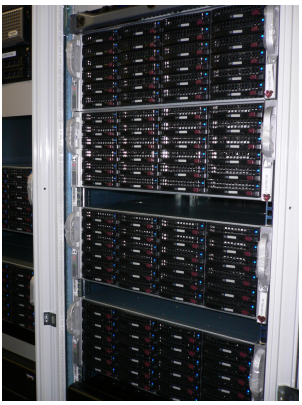

- The event tags are determined by the trigger algorithms based on the event content

- To facilitate off-line data distribution, every event is written to multiple files, one per each of its tags

- File checksum is calculated while writing $\Rightarrow \mathrm{CPU}$-intensive! 


\section{The current Data Logging system: overview}

\section{Purpose}

5 PCs receive data from the Event Filter system and write it to local disks. Each event is:

- analyzed to determine the "tags" applied by the Event Filter trigger

- processed (e.g. compressed)

- written to appropriate file(s) according to the tags

\section{Details}

- The event tags are determined by the trigger algorithms based on the event content

- To facilitate off-line data distribution, every event is written to multiple files, one per each of its tags

- File checksum is calculated while writing $\Rightarrow \mathrm{CPU}$-intensive! 


\section{The current Data Logging system: limitations}

- The current Data Logger implementation is essentially single-threaded:

- multiple threads receive the events from the EF

- a single thread does the processing and writing

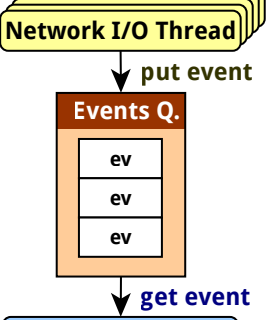

Processing and Writing Thread 


\section{The current Data Logging system: limitations}

- The current Data Logger implementation is essentially single-threaded:

- multiple threads receive the events from the EF

- a single thread does the processing and writing

- This design is very unlikely to scale:

- maximum processing throughput: $500 \mathrm{MB} / \mathrm{s}$

- comparable with I/O (network and disks) limits

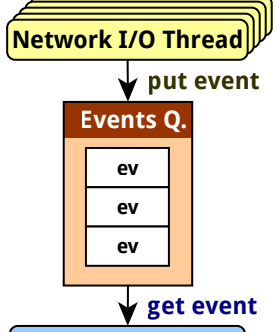

Processing and Writing Thread 


\section{The current Data Logging system: limitations}

- The current Data Logger implementation is essentially single-threaded:

- multiple threads receive the events from the EF

- a single thread does the processing and writing

- This design is very unlikely to scale:

- maximum processing throughput: $500 \mathrm{MB} / \mathrm{s}$

- comparable with I/O (network and disks) limits

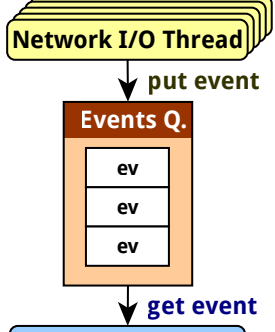

Processing and Writing Thread

- It is a major blocker for the addition of new features requiring more CPU power than a single core can provide

- due to this event-level data compression currently needs to be performed off-line, as an additional step 


\section{New design: general considerations}

- The data processing workload is embarrassingly parallel: the incoming data are already divided in events

The raw data file format is strictly sequential

$\Rightarrow$ It is impossible to do concurrent writes to the same file

- Necessary to calculate the overall file checksum before writing to disk, aiding in the detection of write errors

- Keeps the format complexity to a minimum 


\section{New design: general considerations}

- The data processing workload is embarrassingly parallel: the incoming data are already divided in events

\section{Constraint}

The raw data file format is strictly sequential

$\Rightarrow$ It is impossible to do concurrent writes to the same file

- Necessary to calculate the overall file checksum before writing to disk, aiding in the detection of write errors

- Keeps the format complexity to a minimum 


\section{New design: general considerations}

- The data processing workload is embarrassingly parallel: the incoming data are already divided in events

\section{Constraint}

The raw data file format is strictly sequential

$\Rightarrow$ It is impossible to do concurrent writes to the same file

- Necessary to calculate the overall file checksum before writing to disk, aiding in the detection of write errors

- Keeps the format complexity to a minimum

- Multiple events can be written to different raw data files concurrently, but no more than one event can be written to each data file at once 


\section{New design: idea}

- Split the workload in tasks

- For each event:

- one task does the processing

- multiple tasks do the writing

(for each tag, a task writes the event to the corresponding file). 


\section{New design: idea}

- Split the workload in tasks

- For each event:

- one task does the processing

- multiple tasks do the writing (for each tag, a task writes the event to the corresponding file).

- Use a single thread pool to execute the tasks

- Schedule the tasks cleverly to avoid locking 


\section{New design: idea}

- Split the workload in tasks

- For each event:

- one task does the processing

- multiple tasks do the writing (for each tag, a task writes the event to the corresponding file).

- Use a single thread pool to execute the tasks

- Schedule the tasks cleverly to avoid locking

- At any given time:

- any number of processing tasks can run

- for each raw data file, only one task writing to it can run 


\section{New design: finally, a diagram!}

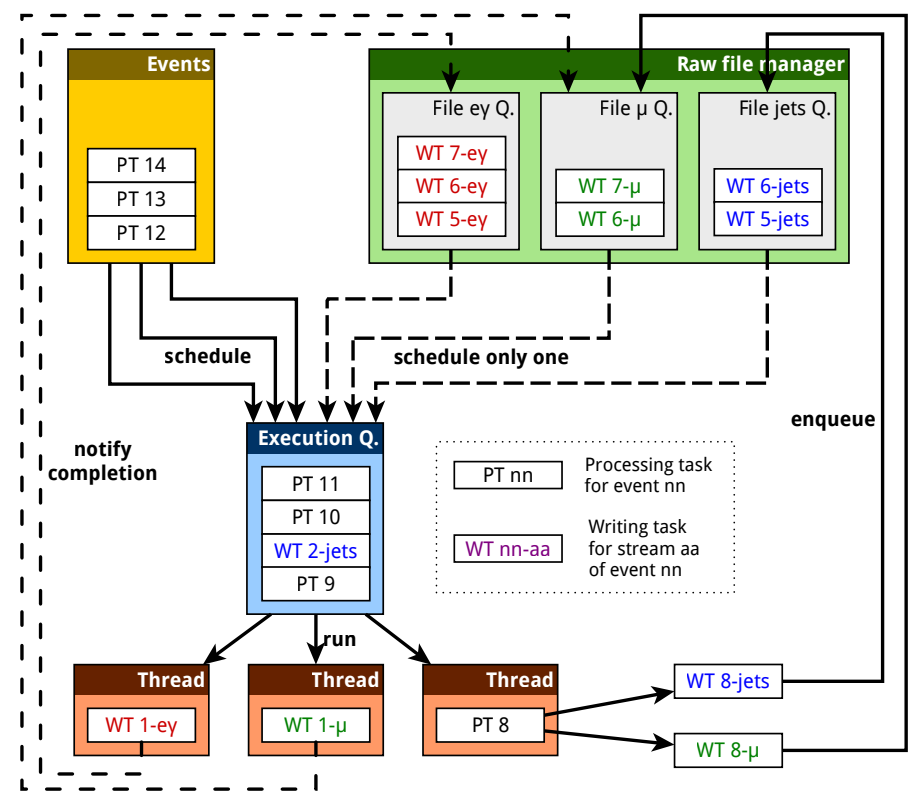




\section{New design: implementation with Threading Building Blocks}

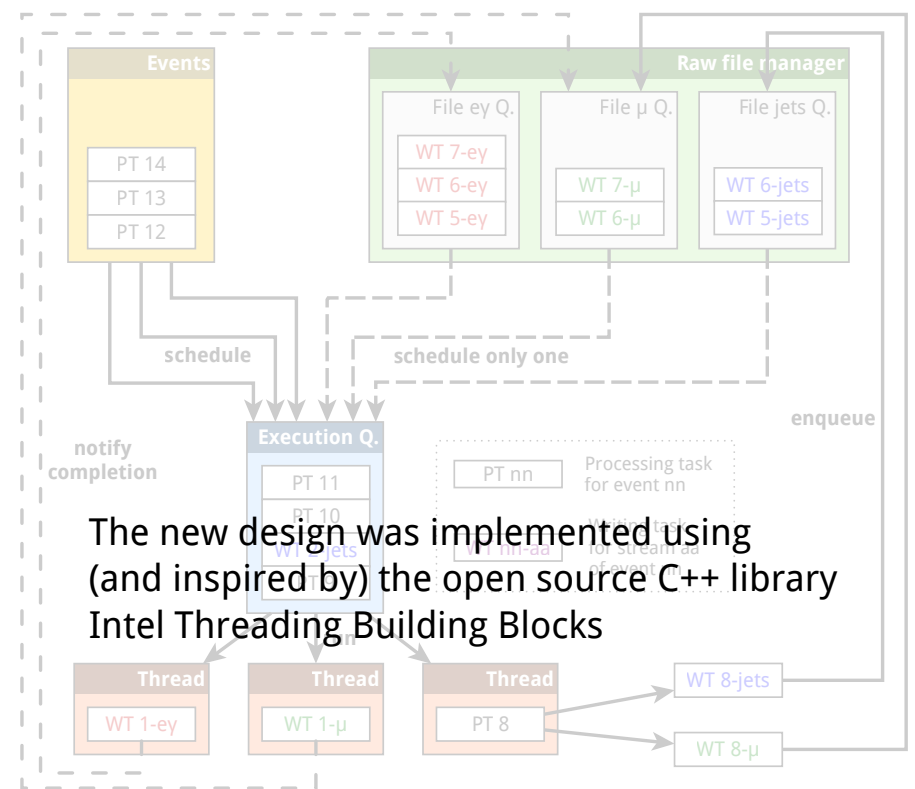




\section{New design: implementation with Threading Building Blocks}

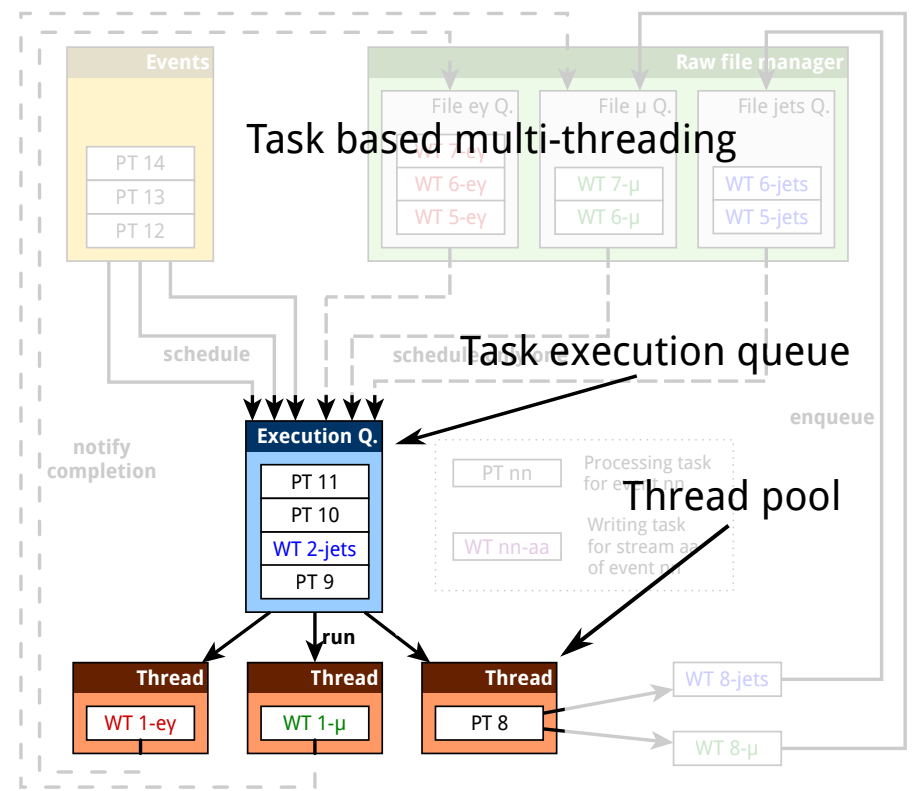




\section{New design: implementation with Threading Building Blocks}

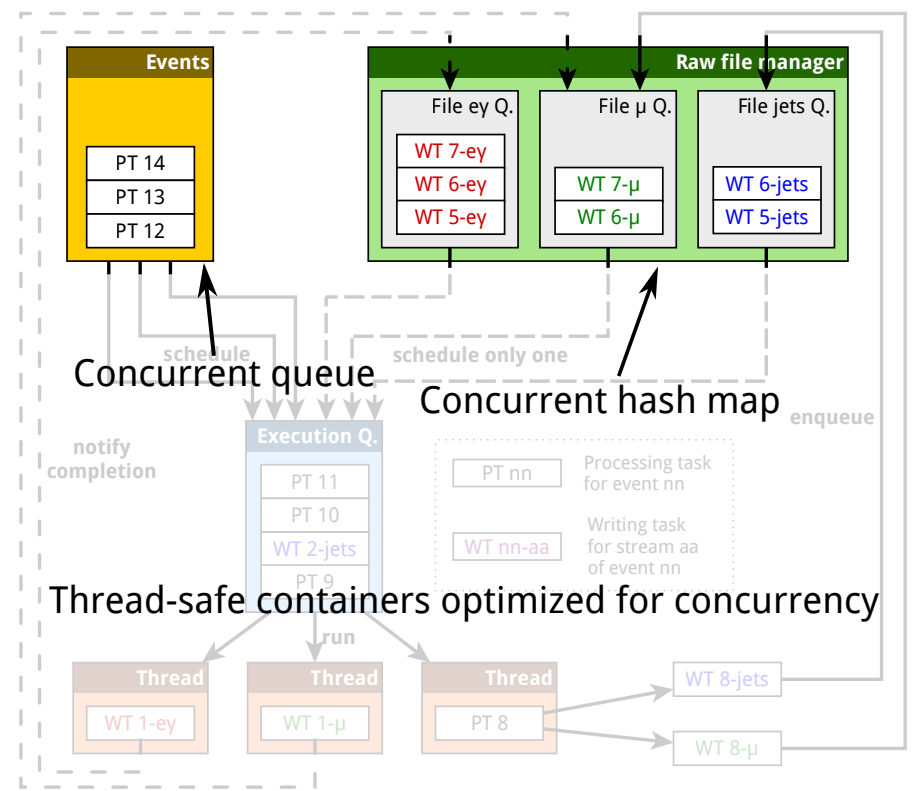




\section{Performance evaluation: resource utilization}

- The new implementation was tested and compared with the old one

- in the current production system

- in a testbed with older hardware

single Datalogger machine was operated at saturation

- The dataset consisted of actual event data,

with 1 to 4 tags assigned to each event

> changing the number of tags per event

changes the number of files the Data

Logger has to write each event to

> does not change the required network

bandwidth
Testbed Data Logger PC

\begin{tabular}{|c|}
\hline 2x dual-core Xeon 5130 \\
\hline 4 GB RAM \\
\hline $3 x$ 3ware RAID5 array \\
\hline $2 x$ GbE NIC \\
\hline
\end{tabular}

\section{Production Data Logger PC}

\begin{tabular}{|c|}
\hline 2x quad-core Xeon E5520 \\
\hline 24 GB RAM \\
\hline 3x Adaptec RAID5 array \\
\hline $2 x$ GbE NIC \\
\hline
\end{tabular}




\section{Performance evaluation: resource utilization}

- The new implementation was tested and compared with the old one

- in the current production system

- in a testbed with older hardware

- A single Data Logger machine was operated at saturation

- The dataset consisted of actual event data,

with 1 to 4 tags assigned to each event

> changing the number of tags per event

changes the number of files the Data

Logger has to write each event to

- does not change the required network

bandwidth 


\section{Performance evaluation: resource utilization}

- The new implementation was tested and compared with the old one

- in the current production system

- in a testbed with older hardware

- A single Data Logger machine was operated at saturation

- The dataset consisted of actual event data, with 1 to 4 tags assigned to each event

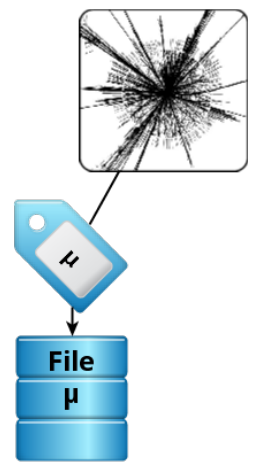

- changing the number of tags per event changes the number of files the Data Logger has to write each event to

- does not change the required network bandwidth 


\section{Performance evaluation: resource utilization}

- The new implementation was tested and compared with the old one

- in the current production system

- in a testbed with older hardware

- A single Data Logger machine was operated at saturation

- The dataset consisted of actual event data, with 1 to 4 tags assigned to each event

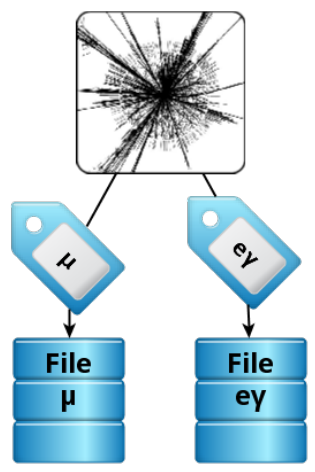

- changing the number of tags per event changes the number of files the Data Logger has to write each event to

- does not change the required network bandwidth 


\section{Performance evaluation: resource utilization}

- The new implementation was tested and compared with the old one

- in the current production system

- in a testbed with older hardware

- A single Data Logger machine was operated at saturation

- The dataset consisted of actual event data, with 1 to 4 tags assigned to each event

- changing the number of tags per event changes the number of files the Data Logger has to write each event to

- does not change the required network bandwidth

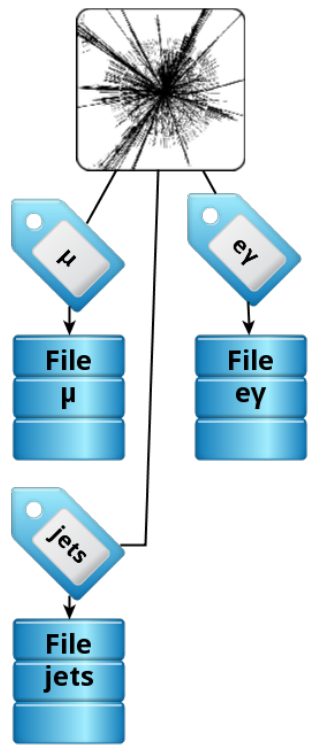




\section{Performance evaluation: resource utilization}

- The new implementation was tested and compared with the old one

- in the current production system

- in a testbed with older hardware

- A single Data Logger machine was operated at saturation

- The dataset consisted of actual event data, with 1 to 4 tags assigned to each event

- changing the number of tags per event changes the number of files the Data Logger has to write each event to

- does not change the required network bandwidth

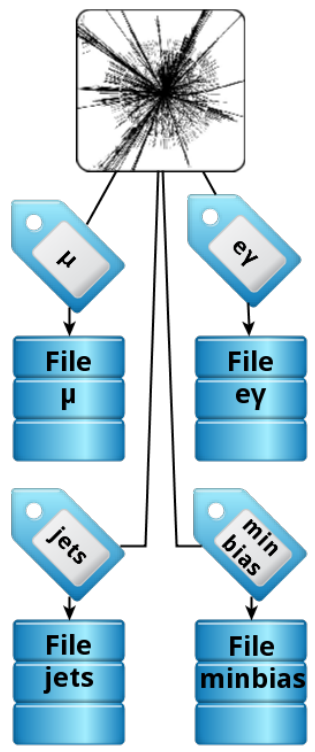




\section{Performance evaluation: resource utilization}

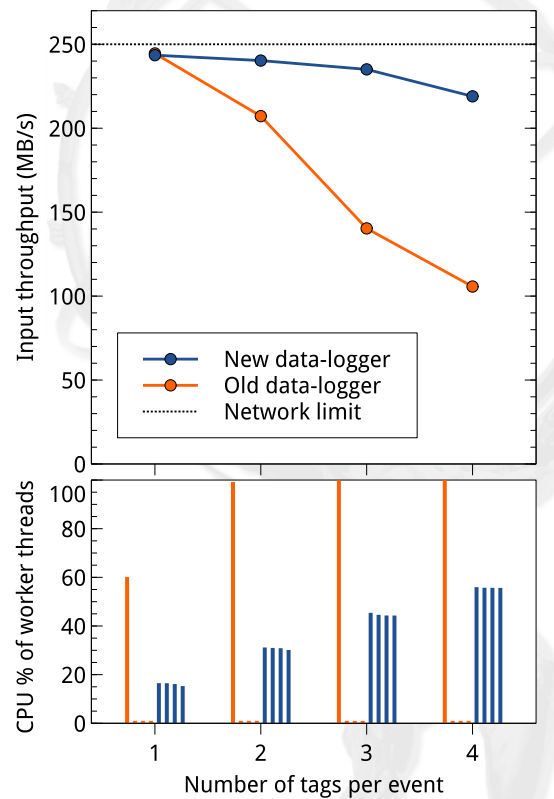

The "hard" limit on the throughput of a single Data Logger is given by the network bandwidth: $2 \mathrm{~Gb} / \mathrm{s} \simeq 250 \mathrm{MB} / \mathrm{s}$

Old single-threaded implementation

- Can operate at network saturation only for a single tag per event

- Above 2 tags per event, the load generated by its single thread exceeds what a single CPU core can take

- The throughput decreases accordingly 


\section{Performance evaluation: resource utilization}

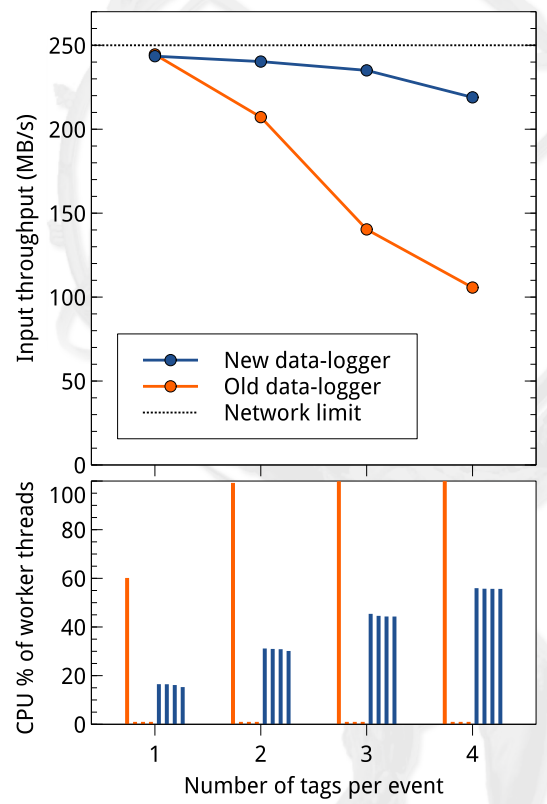

The "hard" limit on the throughput of a single Data Logger is given by the network bandwidth: $2 \mathrm{~Gb} / \mathrm{s} \simeq 250 \mathrm{MB} / \mathrm{s}$

New multi-threaded implementation

- The throughput is almost unaffected by the load

- Its 4 threads spread the workload on the 4 CPU cores: none of them uses more than $60 \%$ of a core

- Leaves plenty of headroom for additional CPU intensive processing $\Rightarrow$ compression 


\section{Performance evaluation: scalability (in testbed)}

- On-line event compression (with zlib) radically changes the landscape

- The time spent compressing events ( 50 ms per $\mathrm{MB}$ ) dominates the rest of the processing ( 2 ms per $\mathrm{MB})$

- throughput is much lower

- all workloads saturate the CPU

- One can examine the throughput as a function of the number of CPU cores (threads) used

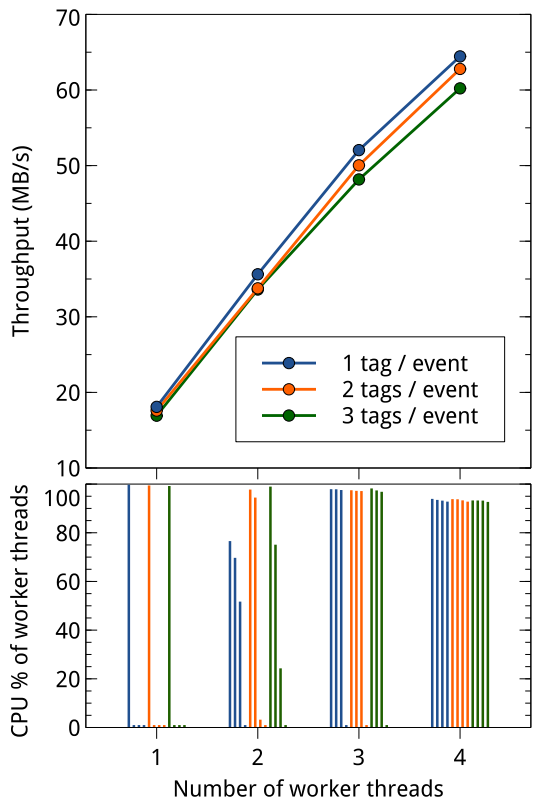




\section{Performance evaluation: scalability (in testbed)}

- On-line event compression (with zlib) radically changes the landscape

- The time spent compressing events ( 50 ms per MB) dominates the rest of the processing ( 2 ms per $\mathrm{MB})$

- throughput is much lower

- all workloads saturate the CPU

- One can examine the throughput as a function of the number of CPU cores (threads) used

- scaling is (almost) linear

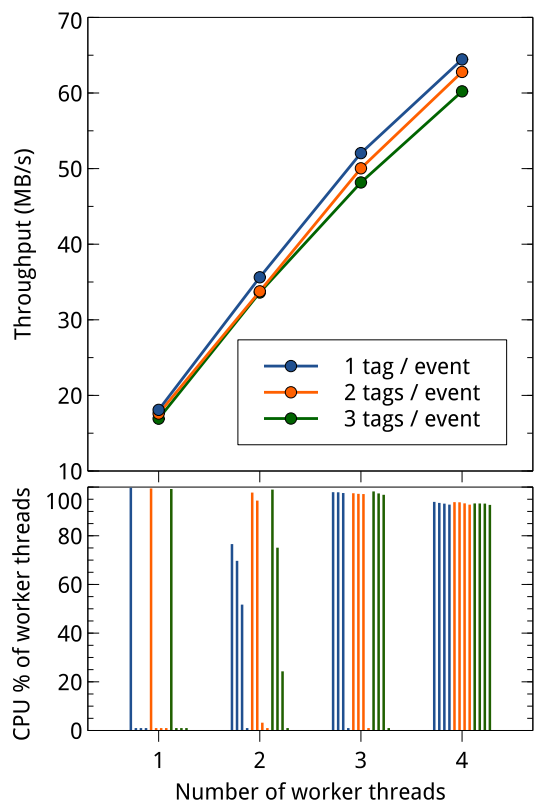




\section{Performance evaluation: scalability (in production)}

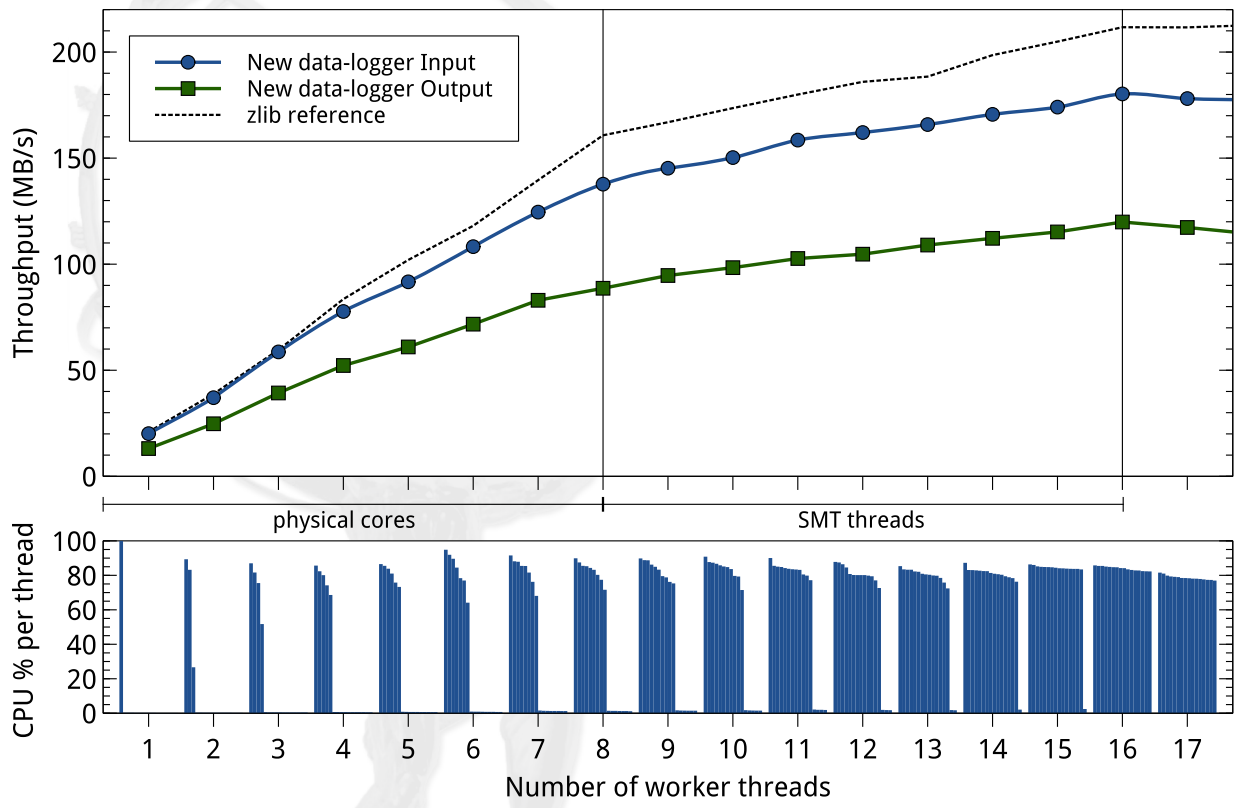




\section{Performance evaluation: scalability (in production)}

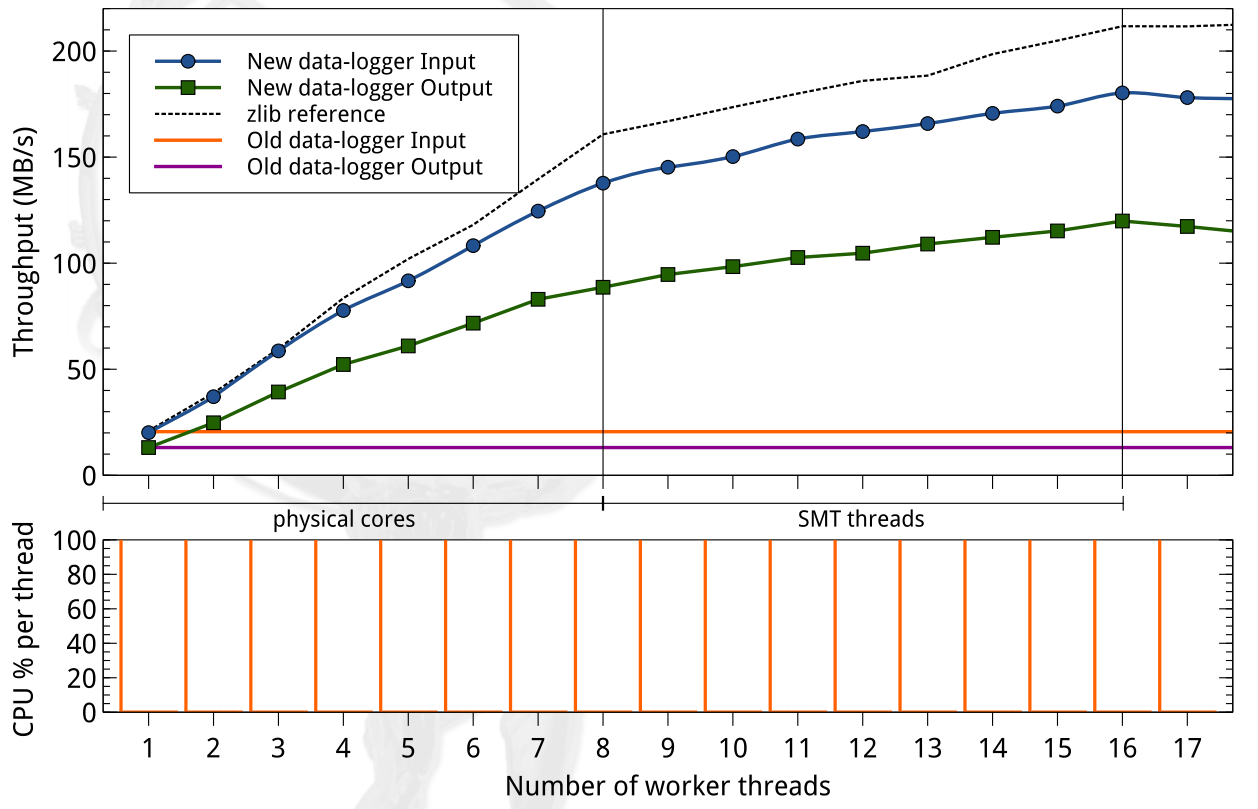




\section{Conclusions}

- A novel design for the ATLAS Data Logging application was implemented and thoroughly tested

The performance of the new software is very satisfactory:

taps into the full power of modern CPUs

- future-proofs the Data Logger

enables the addition of computationally-intensive features

- It will be one of the essential components of

the "evolved" system currently being

developed to meet the challenges of $\mathrm{LHC}$

data-taking in 2014 and beyond

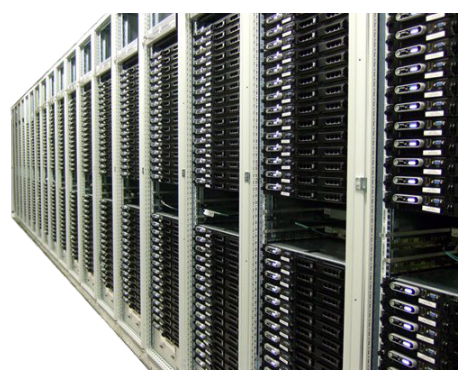




\section{Conclusions}

- A novel design for the ATLAS Data Logging application was implemented and thoroughly tested

- The performance of the new software is very satisfactory:

- taps into the full power of modern CPUs

- future-proofs the Data Logger

- enables the addition of computationally-intensive features

- It will be one of the essential components of the "evolved" system currently being developed to meet the challenges of $\mathrm{LHC}$ data-taking in 2014 and beyond
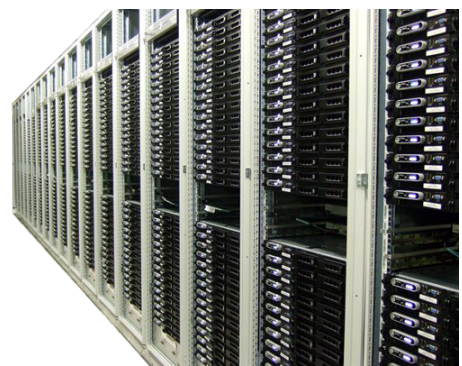


\section{Conclusions}

- A novel design for the ATLAS Data Logging application was implemented and thoroughly tested

- The performance of the new software is very satisfactory:

- taps into the full power of modern CPUs

- future-proofs the Data Logger

- enables the addition of computationally-intensive features

- It will be one of the essential components of the "evolved" system currently being developed to meet the challenges of LHC data-taking in 2014 and beyond

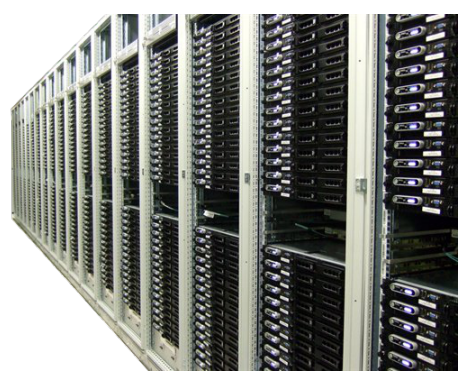




\section{Backup}




\section{Other design constraints}

Operations are driven by the received event data

The Data Logger can only rely on the information it gathers by examining the received events

No assumptions about the data flow

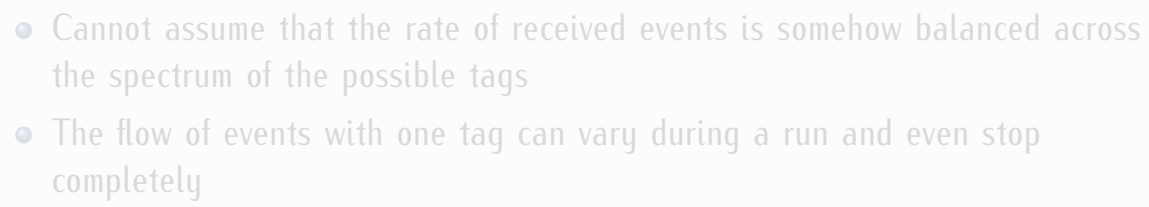




\section{Other design constraints}

Operations are driven by the received event data

The Data Logger can only rely on the information it gathers by examining the received events

No assumptions about the data flow

- Cannot assume that the rate of received events is somehow balanced across the spectrum of the possible tags

- The flow of events with one tag can vary during a run and even stop completely 


\section{Other possible designs: thread pool with locking}

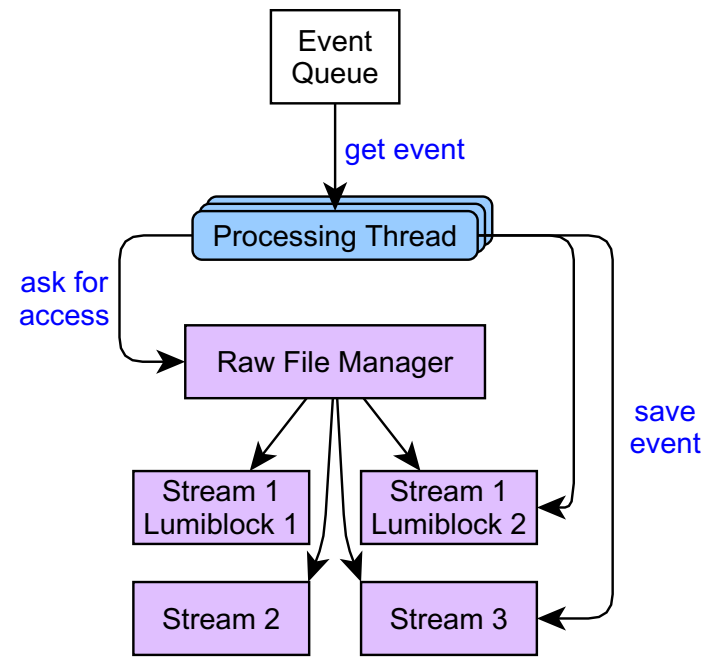




\section{Other possible designs: chain of responsibility}

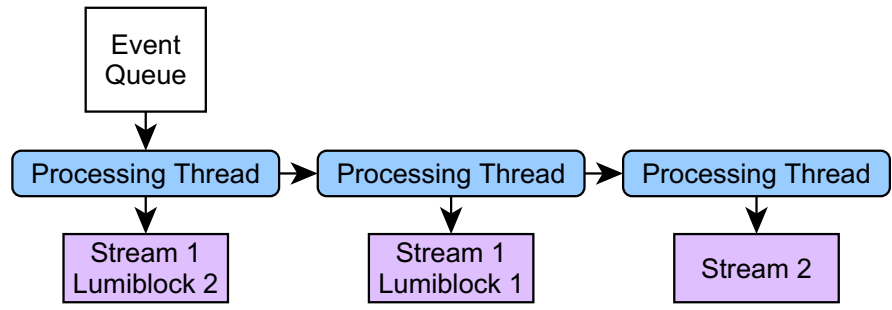

Risk of starvation! 


\section{Other possible designs: one thread pool per file}

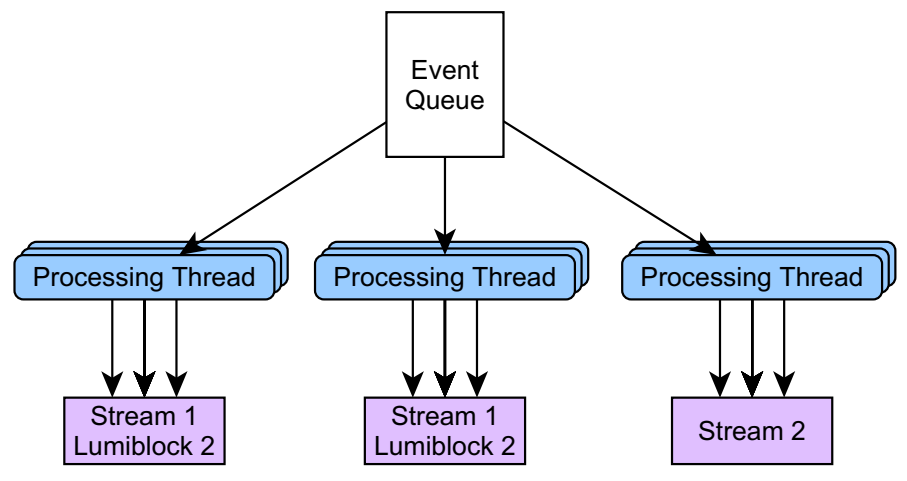

Too many threads! 


\section{zlib performance evaluation}

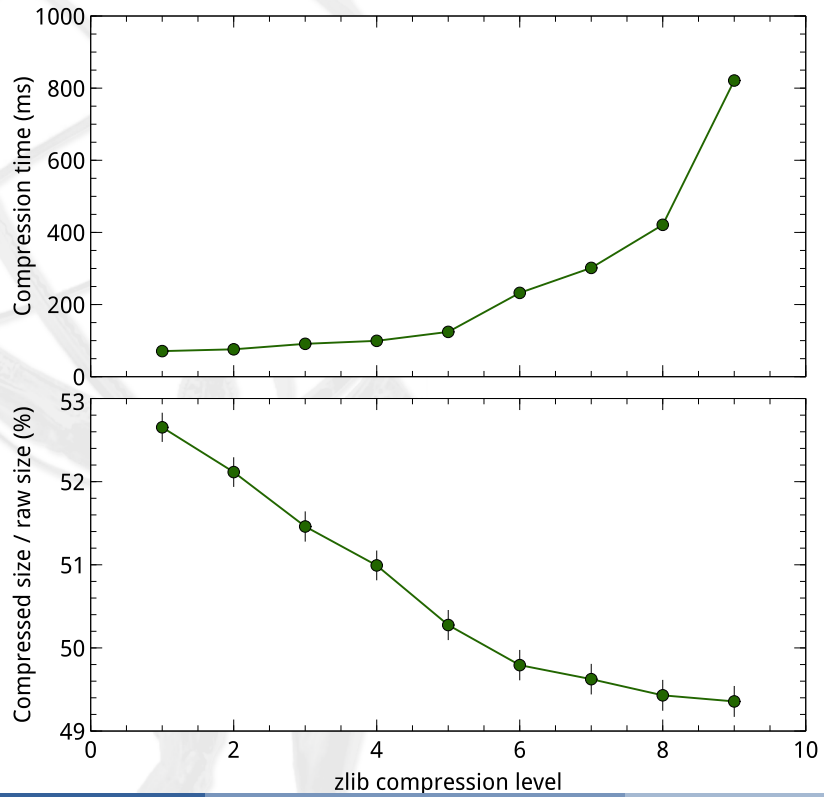

T. Colombo, W. Vandelli (Pavia U., CERN)

ATLAS Highly-parallel Data-Logging System 